\title{
Integrating Voice over Internet Protocol Technology as a Communication Tool on a Converged Network in Laos
}

\author{
Phoutthasine Chouyapaseuth \\ School of Computer Science and Technology, Wuhan University of Technology, Wuhan, China
}

\begin{abstract}
Currently computer network is widely using in transmitting information especially the connection between servers located in Head Quarter and users in branches. Beside the information, they have to call to others which is separated system. If they consolidate into the existing computer network, the organization can deduct some expenses and boost the utilization on existing infrastructures. The VoIP technology has been widely accepted and has received a boost in many countrieswhere users are now migrating from the legendry Public Switch Telephony Network (PSTN) to VoIP because of the numerous benefits it offers but unfortunately the organizationin Laos is yet to key into this promising technology. This study therefore focuses on introducing and implementing this technology in a converged network in Laos environment by the use of open source Asterisk.
\end{abstract}

Keywords: VoIP, SIP, Converged Network, Asterisk.

\section{INTRODUCTION}

VoIP, also known as IP Telephony, is the real-time transmission of voice signals using the Internet Protocol (IP) over the public Internet or a private data network [1]. In simpler terms, VoIP converts the voice signal from your telephone into a digital signal that travels over the Internet [2]. One of the most significant advantages of VoIP over a traditional public switched telephone network (PSTN) is that one can make a long distance phone call and bypass the toll charge.This integrated voice/data solution allows organization to carry voice applications over their existing data networks. Not only will this technological advancement have an impact on the large traditional telecommunications industry, it will alter the pricing and cost structures of traditional telephony. Furthermore, when compared with circuit-switched services, IP networks can carry 5 to 10 times the number of voice calls over the same bandwidth [3]. However, VoIP still suffer few drawbacks which user should consider when deploying VoIP systems. Some of VoIP systems advantages and disadvantages are summarized below.

\section{Advantaged}

- Low cost.

- Flexibility

- Provides voice mail and call forwarding.

- Free services gained usually when connecting from PC to PC.

- Users can make VoIP calls from anywhere for long distance or international calls.

- Easy to implement and install.

- Network capacity utilization.

- Integration with other available services over the Internet.

\section{Disadvantages}

- Users can not make calls during power outages

- Connection limitation to emergency services.

- Depends on Internet connection condition.

- IP network that does not guarantee Quality of Service (QoS) for voice communication

Basically, VoIP systems can be configured in these connection modes respectively; PC to PC, Phone to Phone and $\mathrm{PC}$ to Phone. Moreover, Phone can be digital type or analogue type. In case of analogue phone, it would be connected to the system via adapters which convert the analog signals into digital format.

\section{RESEARCH METHODOLOGY}

When planning to implement VoIP in a converged network, choosing an approach will depend on factors like budget, technology expertise, implementation risk and cost of deployment. In my implementation scenario, VoIP will be implemented between two offices in different location. The Vientiane office which is the Head office and the Thatluang office is branch office. In this case study, an open source Asterisk will be implemented on a converged.

\subsection{VoIP Network Components}

The VoIP systems can come in different forms. Its basic structure is functionally similar to that of PSTN that allows it to communicate with the second party at the other terminal of the connection which is either a VoIP system or traditionalanalog telephone. Its basic form can be grouped into three; [4] 
Vol. 5, Issue 9, September 2016

\subsubsection{End users devices}

The end user devices in a VoIP setup consists of hard phones and soft phones that provides an interface in which voice users interact with other users as well as the system. They use TCP/IP protocol to communicate with IP network that has an IP address for subnet on which they are installed. A soft phone on the other hand runs on software application on computers and also installed on mobile devices and have the same base features as VoIP phones. [5]
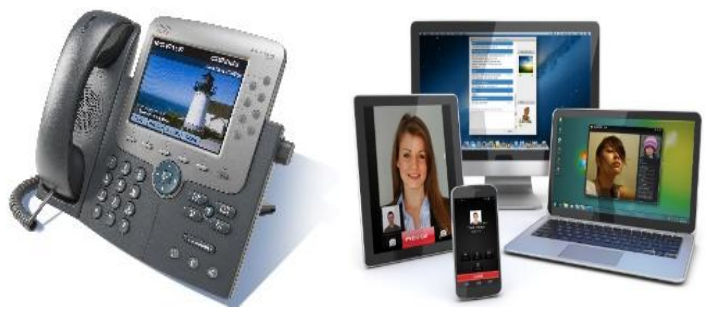

Fig.1 End user devices

\subsubsection{Network Components}

The main component in there is the IP PBX. The IP PBX which simply stands for IP private exchange box is a telephone switching system situated within the enterprise that switches calls between VoIP users on a local line whileenabling users to share some certain number of external phone lines[6]. IP PBX can be used to switch calls between a VoIP and PSTN user just like the conventional PBX does.

It has an advantage of converging data and voice network which provides flexibility and reduced long-term operational andmaintenance cost for an organization. Other network components include switches and routers.

\subsubsection{VoIP Gateway/Gatekeepers}

In VoIP, a gateway is a device that converts voice calls in real time between the PSTN and the IP network [7]. Its main function includes voice marketization, compression/decompression, call routing and control signaling. It may also additionally serve as an interface to external controllers taking the gatekeepers or soft switches, network management system and billing system.

The gatekeeper on the other hand is a centrally controlled entity that performs management functions such as authentication, address mapping and bandwidth management in a VoIP solution for multimedia application such as video conferencing.

The gatekeeper performs other numerous functions like providing intelligence to the system, authorization and authentication service, address resolution and logging of call detail record. The gatekeeper also controls bandwidth, provides interfaces to existing legal system and monitors network for engineering purpose.

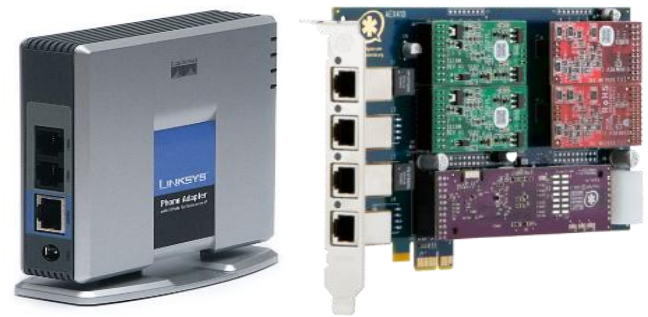

Fig.2 VoIP Gateway

\subsection{Detail Design}

The project basically consists of 2 different networks with two Asterisk server placed in Head office and Branch office network and Asterisk server using SIP. Phones with extension 1XXX and 2XXX are registered to two Asterisk server. However RTP is the actual protocol to transfer real-time voice data between clients and server in collaboration with SIP which is used for signaling between clients and server. For the connection between two networks, SIPis used as a trunk to transfer both signaling and real-time voice data. G711 is codec types that is given permission to be transmittedwithin the particular protocol (SIP) defined.

Table 1: IP Addressing and Extension Allocation

\begin{tabular}{|c|c|c|}
\hline Description & Head Office & Branch Office \\
\hline $\begin{array}{c}\text { IP } \\
\text { addressfor } \\
\text { VoIP } \\
\text { Network }\end{array}$ & $192.168 .2 .0 / 24$ & $192.168 .2 .0 / 24$ \\
\hline $\begin{array}{c}\text { Extension } \\
\text { Numbers }\end{array}$ & $2 \mathrm{XXX}$ & $1 \mathrm{XXX}$ \\
\hline $\begin{array}{c}\text { IP } \\
\text { PBXassigne } \\
\text { d IP } \\
\text { address }\end{array}$ & 192.168 .2 .200 & 192.168 .2 .201 \\
& & 255.255 .255 .0 \\
\hline
\end{tabular}

\subsection{Asterisk}

Asterisk is an open source framework for building communications applications, it was created in 1999 by Mark Spencer of Digium[8].Asterisk turns an ordinary computer into a communications server. Asterisk powers IP PBX systems, VoIP gateways, conference servers and other custom solutions. It is used by small businesses, large businesses, call centers, carriers and government agencies, worldwide. Asterisk is free and open source. Because it can run conveniently on mid-range PC hardware and its open nature as compared with other commercial VoIP deployment, it has become a choice of VoIP users. Asterisk runs on Linux platform and was released under GNU General Public License.[9]

Asterisk runs on UNIX operating system like Linux, Mac OS X, OpenBSD, FreeBSD and Sun Solaris and can be accessedthrough the command line interface (CLI). Various version ofAsterisk has been developed after it was released in 1999with the latest being Asterisk 
Vol. 5, Issue 9, September 2016

13.11.2released in Sep 9, 2016. It hasmore than 200 notable new features which include newsecurity features, more than 200 enhancements, integrationwith IPv6 and lots more Asterisk supports protocol like SIP,IAX, H323, MGCP and SCCP[10] but SIP protocol was usedin this implementation.

\subsubsection{Configuring Asterisk}

In configuring Asterisk, two different approaches can be deployed; these can be either configuration method by manually editing or the GUI using point and click. The manual editing was used for this implementation. During the configuration process.

Sip. Conf: In this file the various sip clients were defined in their context for the Asterisk PBX, the command used is:\#nano /etc/asterisk/sip.conf

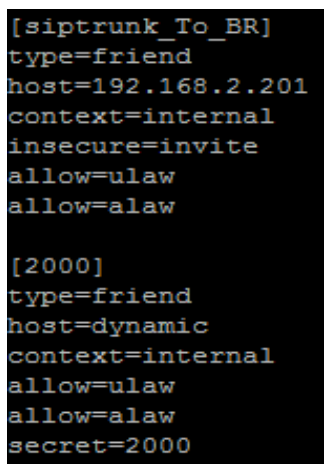

Fig.3sip dial Plan on Head Office

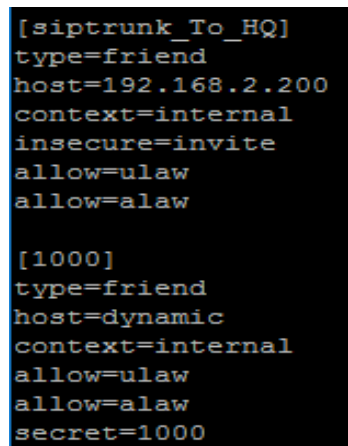

Fig.4sip dial plan on Branch Office

Extensions. Conf: This file defines how calls are handled and routed within and outside the Asterisk box. The dial plan for various users is also defined in their context that determineshow they interact in the Asterisk, the command used is:

\#nano /etc/asterisk/extensions.conf

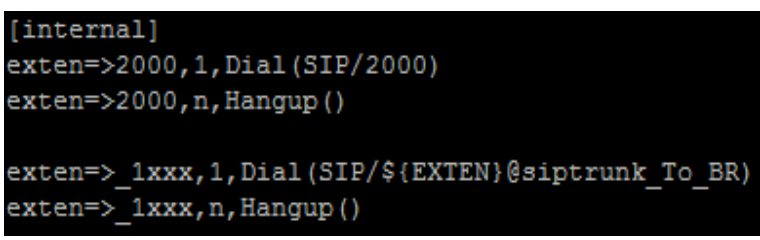

Fig.5extension dial plan on Head Office

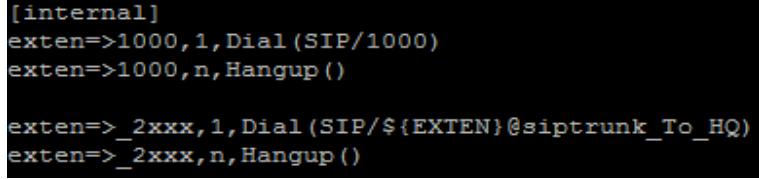

Fig.6extension dial plan on Branch Office

To check the status of the configured users, its extension number, IP address, port number and status, the command used is:>sip show peers

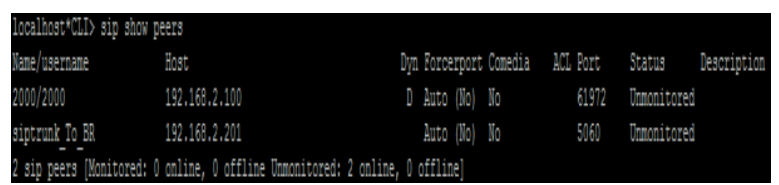

Fig.7the status of the configured users

After the configured user's status was checked, a call was place between the two networks using both the TraditionalTelephones and the soft pones. The call was initiated and received successfully.

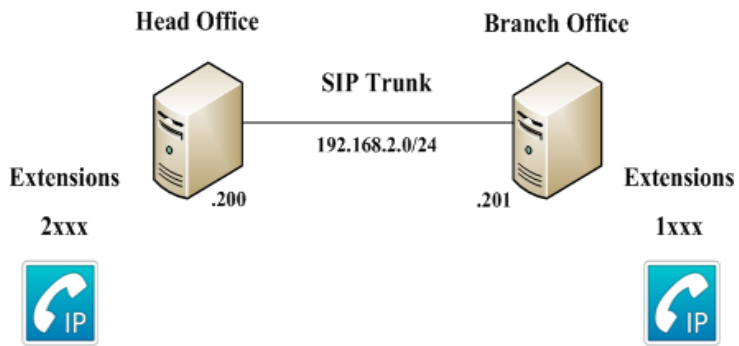

Fig.8 VoIP network

\section{Comparison of VoIP and PSTN Services}

To comparing the VoIP technology and the legendary PSTN, a table that compares the properties of each of this technology is below.

\section{VoIP Services}

- Packet Switched. No reservation of bandwidth, network resources are not used when packets are not transmitted.

- Uses SIP, H323, RTP and several other transmission protocol to transmit data

- Provides almost all traditional service and others like video, data and multimedia services

- No bandwidth reservation and the quality can be affected by high traffic but quality can also be better than the PSTN with sufficient bandwidth

- Both data and voice share the same infrastructure

- No independent power supply, alternative power is normally arranged.

- Open architecture with almost no restriction

- No built emergency mechanism.

- Unsecured due to its open nature

- Relatively low as existing data network can be used for transmitting voice 
Vol. 5, Issue 9, September 2016

\section{PSTN Services}

- Circuit Switched. Band width are used here even when information is not being transmitted

- Uses SS7 signal protocol

- Traditional services like Phone calls, voice mail box, faxes, caller ID etc

- Quality here is guaranteed based on $64 \mathrm{kbps}$ bandwidth reservation

- Segmentation of infrastructure is necessary

- Telephone lines transmit power of $48 \mathrm{~V}$ which the Telephone uses even during power outage.

- Access is limited

- Caller may be localized during the case of an emergency as each client has their subscriber line.

- Reasonably secured

- Cost is High due to additional infrastructure and management

It can be observed from comparison that the VoIP technology is still undergoing some improvement especially in terms of quality of service and security. Different QoS method has been developed and implemented that will prioritize real time traffic like the voice and video over the data traffic to grantee quality delivery of packets. The importance of security on the VoIP network cannot be overemphasized.

All VoIP users require Confidentiality, Integrity and Availability (CIA) when this technology isbeing put to use. This two major factor has been looked into, with the major improvement on security being the encryption of voice packet over both public and private network. One major attraction to VoIP technology when compared to the PSTN is the reduced cost and the other several add-ons it offers.

\section{CONCLUSION}

The VoIP technology has been predicted to be the future to telecommunications globally; therefore, integrating it in a converged network in Laos must be encouraged. The VoIP technology has cheaper call rate, easier IT management and reduction in operational cost for a combined network for voice and data which gives it an edge over the PSTN.

It will only be considered as being successful and replace the traditional PSTN when both the security and quality of service of the voice packet which is real-time transmitted over the public and private network is specially addressed. This technology when implemented by organization in Laos over the already existing infrastructureswith adequate bandwidth and security will go a long way in easing communication.

\section{REFERENCES}

[1] SheetalJalendry, ShradhaVerma. 2015. A Detail Review on Voice over Internet Protocol (VoIP). International Journal of Engineering Trends and Technology (IJETT) - vol.23 (4), pp. 161-166.
[2] AnestisPapakotoulas. 2014. Voice over Internet Protocol. Journal of Computations \& Modelling. vol.4 (1), pp. 299-310.

[3] M.A. Mohamed, F.W. Zaki and A.M. Elfeki. 2012. Performance Analysis of VoIP Codecs over WiMAX Networks. IJCSI International Journal of Computer Science Issues, Vol. 9, Issue 6, No 3, November 2012

[4] Dantu,R., Fahmy, S., Schulzrinne, H., \&Cangussu, J.(2009), 'issues and challenges in security VoIP'Computer\& Security,28(8), pp .743-753.

[5] Desantis,M. (2008), Understanding Voice over Internet Protocol (VoIP), US-CERT.

[6] Ramachandran, (2006) VoIP Security: asserting thetrust boundary, 'The Global Voice of InformationSecurity', ISSA Journal pp.8-13.

[7] Dhamankar, R. (2005), Intrusion Prevention: The Future of VoIP Security, White paper by Tripping Point.

[8] Asterisk the Open Source PBX. http://www.asterisk.org/ [April 3, 2016]

[9]. Russell Bryant, Leif Madsen, and Jim Van Meggelen. Asterisk ${ }^{\text {TM: }}$ The Definitive Guide, Fourth Edition. Copyright (C) 2013, O'Reilly Media, Inc. All rights reserved. Printed in the United States of America. ISBN: 978-1-449-33242-6.

[10] www.digium.com/en [assessed 20th Sep, 2016] 